\title{
Five points on columns
}

\section{Kathleen S. Rockland*}

Picower Institute for Learning and Memory, RIKEN-MIT Center for Neural Circuit Genetics, MIT, Cambridge, MA, USA

Edited by:

Javier DeFelipe, Cajal Institute, Spain

Reviewed by:

Nick Swindale, The University of British

Columbia, Canada

Javier DeFelipe, Cajal Institute, Spain

*Correspondence:

Kathleen S. Rockland, Picower Institute for Learning and Memory, RIKEN-MIT Center for Neural Circuit Genetics,

Massachusetts Institute of Technology,

77 Massachusetts Ave, Building 46,

Cambridge, MA 02139, USA.

e-mail:kathrock@mit.edu
"Column," like "gene," has both conceptual and linguistic shortcomings. The simple question "what is a column" is not easy to answer and the word itself is not easy to replace. In the present article, I have selected five points, in no way comprehensive or canonical, but which may nevertheless serve as a prompt and aid for further discussions and re-evaluation. These are: that anatomical columns are not solid structures, that they are part of locally interdigitating systems, that any delimited column also participates in a widely distributed network, that columns are not an obligatory cortical feature, and that columns (as "modules") occur widely in the brain in non-cortical structures. I focus on the larger scale macrocolumns, mainly from an anatomical perspective. My position is that cortical organization is inherently dynamic and likely to incorporate multiple processing styles. One can speculate that the distributed mappings within areas like piriform cortex may resemble at least one mode of neocortical processing strategy.

Keywords: collateralization, cytochrome oxidase, layer 2 honeycomb, modularity, terminal arbors, thalamocortical modules, zinc-positive connections

\section{INTRODUCTION}

The column as basic unit and defining cortical attribute has been a compelling, not to say seductive idea. Thus, "column" has persisted both conceptually and linguistically, despite significant problems in both domains. (1) "Column" is ambiguous. It can refer to smallscale minicolumns (diameter $\sim 50 \mu \mathrm{m}$ ), to larger scale macrocolumns (diameter $\sim 300-500 \mu \mathrm{m}$ ), and to multiple different structures within both categories (Jones 2000; Rockland and Ichinohe, 2004; DeFelipe, 2005; Horton and Adams, 2005). It can refer to a functional or to an anatomical grouping. (2) "Column" invites over-simplification. Few if any structures extend from pia to white matter, or even from layer 2 to layer 6; and few if any have definable, "solid" borders.

The simple question, what is a column?, however, is not easy to answer and the word itself is not easy to replace. The column hypothesis is often summarized as stating that "the fundamental unit of cortical organization is a group of interconnected neurons that share a certain set of properties and extend vertically through the cortical layers to form a column" (e.g. Krieger et al., 2007). Key defining features have come to be (1) interconnected neurons (2) with common input, (3) common output, and (4) common response properties; but these criteria have remained difficult to prove. On the one hand, there is overwhelming experimental support in favor of vertical organization ("the cortical column"). On the other, the confirmation of strictly "common" input, output, and response properties has proved elusive (Krieger et al., 2007).

In the present article, I will focus on the larger scale macrocolumns, mainly from an anatomical perspective. I will not address the broader issue of whether or how anatomy predicts function, since this seems best postponed until results from the newer techniques, such as optogenetics, can be assimilated. Issues concerning minicolumns have been discussed previously (Jones, 2000; Rockland and Ichinohe, 2004), and will be considered in other articles in this issue. My case will be that "column," as word and concept, has become too rigid, and has lost the ability to convey the complex and dynamic aspects of cortical organization. Something of this view has been proposed as "emergent properties" of columnar organization (Markram, 2008), and is included in the idea of "cortical cloud" of local synaptic connectivity (Horton and Adams, 2005).

In this brief review, I have selected five points toward a critical re-evaluation of columnar organization. In no way comprehensive or canonical, these are simply intended as a prompt and discussion aid: anatomical columns are not solid structures, they form locally interdigitating systems, any delimited column participates in a widely distributed network, columns are not an obligatory cortical feature, and columns (as "modules") occur widely in the brain, in non-cortical structures. I am not distinguishing among columns, patches, or modules, and will mainly continue to use the term "column", having no obvious alternative to offer.

These same five points can be applied to minicolumns. Minicolumns, by any of the various definitions, also are not "solid." Whether there are different types of minicolumns is unknown, although the literature has tended to favor the view that they are fundamentally repetitive and uniform, as opposed to consisting of distinctly interdigitated systems. The literature has also tended to view minicolumns as local structures, not particularly inter-cooperative or influenced by a spatially distributed network. There is often an assumption that minicolumns are an obligatory cortical feature, although variability across areas has been documented (Peters et al., 1997). That minicolumns are a properly cortical feature is commonly viewed as true, although minicolumn-like dendritic bundles can be found in non-cortical structures (e.g., Roney et al., 1979).

A first section gives background, with a bias toward structural aspects. This is necessarily brief, since a full review is beyond the scope of the present article.

\section{BACKGROUND}

"Macrocolumns" include metabolic zones (e.g., zones of heightened cytochrome oxidase levels), connectional columns or patches, and functional or activity columns. Many, but not all systems of anatomical connections show some degree of columnarity. Thalamic 
and cortical terminations, as visualized by various anterograde tracers injected in vivo, are often dramatically columnar in cross section, especially in layer 4 and adjacent layers. By contrast, cortical or thalamic terminations in layer 1 are in fact transcolumnar, typically diverging over several millimeters. Retrogradely labeled cortical projection neurons in layers 3 and 5 are often patchy or columnar; but patchiness has not been reported for corticothalamic or other projection neurons in layers 5 or 6 , even in highly topographic areas. Thus, at the scale of macrocolumns, multiple mappings can be distinguished, only some of which are columnar (Figure 1).

Serial reconstructions or tangential sections, parallel to the pia and layer 1 , reveal that what appear to be columns in cross section have a variety of shapes and sizes. Ocular dominance columns, one of the textbook examples of columnar organization, are actually slab-like domains; and column width is variable as a function of the visual field; that is, larger in the foveal representation. In the peripheral visual field representation, the slab-like configuration breaks up into patches (Adams et al., 2007). Size and shape variability has been documented across species, and also across individuals within one species (Horton and Hocking, 1996; Adams et al., 2007). Similarly, the barrels in rodent somatosensory cortex are not stereotyped. Hollow barrels, with cell sparse cores, are typical of mice, young rats, and the anterolateral subfield of mature rats, but solid columns, with cell dense cores, are typical of the main posteromedial field in rats (Rice, 1995). Variability is not reported for other columnar systems of connections, but this is likely because many of the systems are harder to visualize globally or require specialized tissue processing.

In primary sensory cortex, stimulation of an eye (in primates) or whisker (in rodents) results in distinct functional ("activity") columns, conspicuously vertical through the cortical layers. These are associated with thalamocortical terminations, but it is important to recognize that the relationship is not direct. First, thalamocortical terminations are layer specific and targeted mainly to layer 4. Other layers receive thalamic terminations, but none of these extend throughout the cortical depth. Consequently, activity columns result from a mix of direct thalamocortical terminations and subsequent intrinsic, intra- and inter-laminar processing (Sato et al., 2007, among others). Secondly, terminal arbors of individual thalamocortical axons are often smaller than the cross-sectional width of activity columns (in monkey: Blasdel and Lund, 1983; Freund et al., 1989). Thus, activity columns result from several factors, both molecular and activity-related (Inan and Crair, 2007), which bring about the convergence of smaller arbors in a 300$500 \mu \mathrm{m}$ wide space.

In association cortices, activity columns are more difficult to visualize and their identification tends to be based on electrophysiological criteria (in primate inferotemporal cortex: Tanaka, 2003). There have been no reports so far of patchy thalamocortical connections to higher order association areas; and the anatomical substrate of functional columns is usually attributed to extrinsic and/or intrinsic cortical connections. These often do have a patchy distribution. In cross section, anterogradely labeled cortical terminations can have a dramatically through-layer columnar appearance. (e.g., autoradiography images in Rockland and Pandya, 1979; Selemon and Goldman-Rakic, 1988). These images, how-
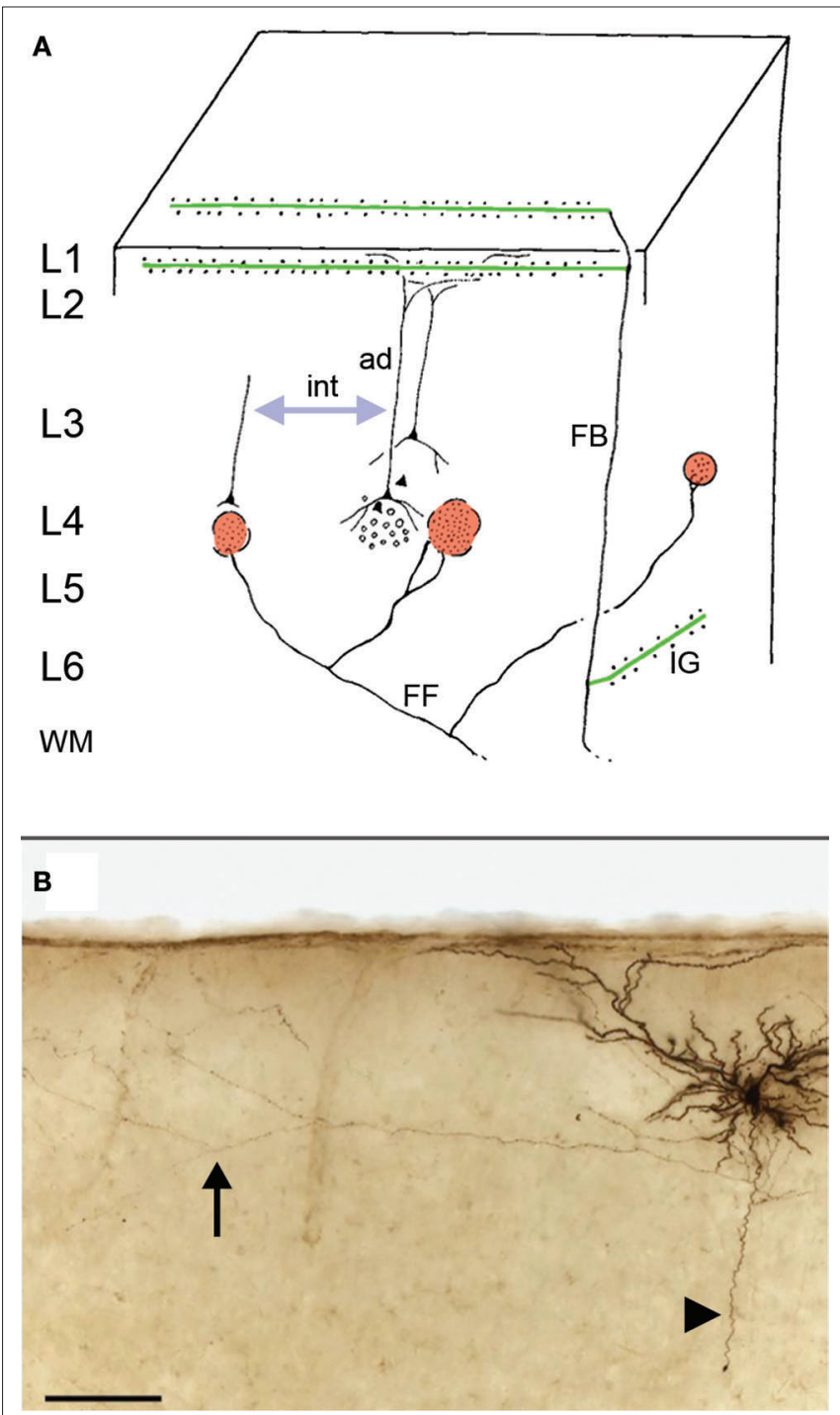

FIGURE 1 | Connectional building blocks: feedforward (FF), feedback (FB), and intrinsic (int) connections. (A) Feedforward (red) and intrinsic (blue arrow) connections are both modular ("columnar"). Pyramidal neurons postsynaptic to feedforward connections presumably are themselves interconnected, but how these extrinsic and intrinsic connections interact is poorly understood. Feedback connections (green) are typically divergent, presumably crossing over a territory corresponding to multiple columns. Modified from Rockland and Drash (1996).

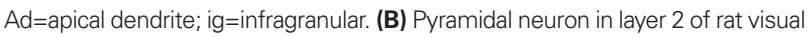
cortex (intracellularly filled with biocytin in vitro). Arrowhead indicates descending axon (truncated in the slice preparation), and vertical arrow points to distal part of an intrinsic axon collateral in layer 2, $600 \mu \mathrm{m}$ from the cell body (courtesy of Dr. Tohru Kurotani). Scale bar $=100 \mu \mathrm{m}$.

ever, can be deceptive. The deeper layer component, as shown by higher resolution Golgi-like markers, may be predominantly axons rather than terminations; and single axon analysis has shown that a terminal patch is composed of individual arbors that are not stereotyped, but rather have different size, shape, and number of terminal boutons (Rockland, 2002).

Cross-sectional cortical columns line up as a series of discontinuous stripes (prefrontal cortex: Pucak et al., 1996). In auditory cortex, both callosal and ipsilateral cortical connections have a com- 
plex configuration in relation to tonotopic organization (Imig and Reale, 1981). Overall, however, compared with thalamocortical terminations, less information is available concerning the size, shape, substructure, and variability of cortical columns; and detailed data concerning their interconnectivity are still lacking.

\section{FIVE POINTS ABOUT STRUCTURAL MACROCOLUMNS POINT 1}

Columns are not solid structures (Figure 2). First, dendrites crossapparent borders in both directions. Pyramidal neurons within a defined column have basal dendrites, oblique dendrites, and apical tufts that extend beyond the home column, and conversely, there will be invading dendrites from neurons in adjoining columns (also: DeFelipe, 2005). In rodent barrel cortex, dendrites of neurons in layer 4 conform to barrel limits (Harris and Woolsey, 1979), but this seems to be an exceptional case. Neurons in layer 4 of primate visual cortex do not comparably conform to ocular dominance boundaries (Katz et al., 1989), and pyramidal neurons in layer 3 extend their dendrites independently of the patches defined by cytochrome oxidase (Hubener and Boltz, 1992; Malach, 1994). This means that some proportion of synapses will contact dendrites whose soma is internal or external to the column, but the actual proportion is not known. Still to be determined as well, is whether contacts are specifically targeted or "randomly" distributed among postsynaptic populations. An interesting question then becomes, how do functionally distinct columns result from an underlying "messy" anatomy.

Second, columns have substructure. In rodent sensory cortex, 2-3 sub-barrel domains have been described on the basis of cytochrome oxidase inhomogeneities and local enrichment of thalamocortical terminations (Land and Erickson, 2005). Similarly,

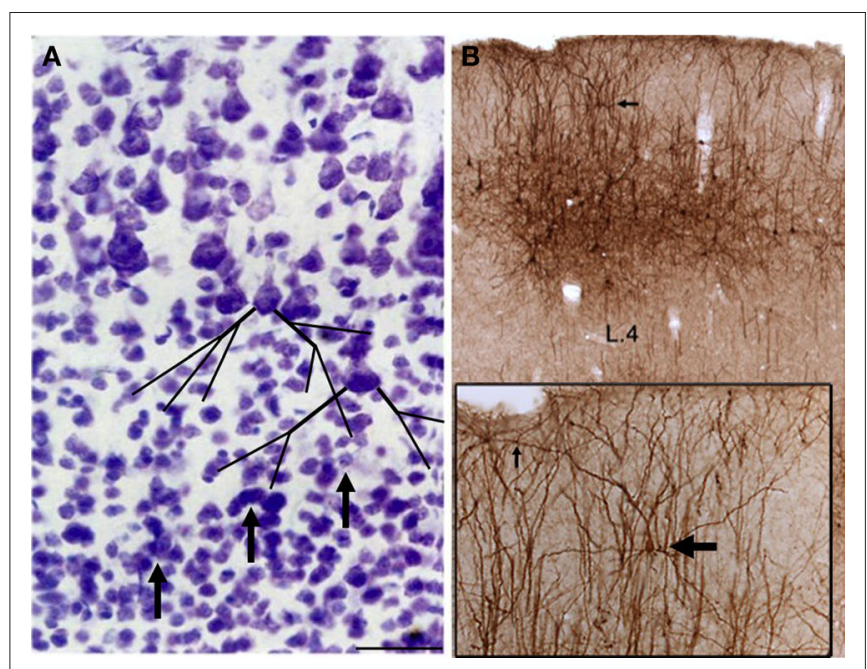

FIGURE 2 | Columns are not solid structures. (A) Cell stain of layer 4 and adjacent layer 3 (macaque temporal association cortex; coronal section). Three distinct cellular rows are apparent in layer 4 (arrows). However, these will be interpenetrated by dendritic and axonal neutrophil; for example, basal dendrites of layer 3 pyramidal neurons, as drawn schematically. (B) A large patch of neurons in anterior temporal cortex, retrogradely labeled by an injection of EGFP-adenovirus (immunoreacted for DAB) in posterior temporal cortex. A layer 2 neuron (horizontal arrow) has a laterally divergent apical dendrite (vertical arrow) extending over $250 \mu \mathrm{m}$ from the soma. Scale bar $=40 \mu \mathrm{m}$ in (A), $500 \mu \mathrm{m}$ for (B), and $100 \mu \mathrm{m}$ for inset. for ocular dominance columns of primate visual cortex, classical anatomical and physiological studies identified core and edge regions, functionally distinguished by different degrees of monocular bias (LeVay et al., 1975). More recently, different conditions of visual deprivation have revealed functional sub-compartments within ocular dominance columns, visualized either by changes in cytochrome oxidase activity (Horton and Hocking, 1998) or by differential expression of immediate-early genes (Takahata et al., 2009).

At the cellular level, there is growing evidence that cortical columns contain multiple, highly specific, fine-scale subcircuits (Yoshimura et al., 2005; Otsuka and Kawaguchi, 2008). This result, which correlates with reports of locally heterogeneous response properties (Sato et al., 2007) weakens arguments in favor of strong intra-columnar homogeneity.

\section{POINT 2}

Columns are partly defined by their neighbors (Figure 3). That is, macrocolumns result from several kinds of interdigitation. This is often considered a mode of parallel processing. It is also related to differential processing by segregated dendritic populations.

\section{Interdigitation of different thalamic nuclei}

The best documented example of two thalamic systems in the same layer is from rodent barrel cortex (Alloway, 2008). In layer 4, thalamocortical projections from the ventral posterior medial or posterior nuclei respectively target the barrels (lemniscal pathway) and their intervening septa (paralemniscal pathway). A similar segregation occurs more generally, but with segregation in different layers. In the primate somatosensory system, calbindin-positive thalamocortical projections terminate in layer 1, and parvalbuminpositive projections terminate in layer 4 (Rausell and Jones, 1991). In this instance, the projections to layer 4 are topographic and patchy, while those to layer 1 are divergent, crossing across multiple columns. In primate visual cortex, divergent thalamic terminations from the lateral geniculate and inferior pulvinar both terminate divergently in layer 1 (Rockland et al., 1999).

\section{Interdigitation of thalamic and cortical systems}

In primary visual cortex of macaques, thalamocortical terminations, visualized as zones of heightened cytochrome oxidase activity, interdigitate with a subset of corticocortical terminations, that contain synaptic zinc, a neuromodulator. This complementarity occurs in layer 4A and in layer 3 (Dyck et al., 2003).

In rat, a similar complementarity has been demonstrated, but in a different laminar location, at the border of layers 1 and 2 . VGLUT-2, a global marker for thalamocortical terminations, forms patches in coronal sections that interdigitate with histochemically reacted zinc, a marker for zinc-positive cortical terminations (Ichinohe et al., 2003). In tangential sections, the patches have a honeycomb or reticular configuration. This is pronounced in rat visual cortex. It is detectable in other areas, at the same superficial level, in both rat and macaque monkey (Ichinohe et al., 2003; Ichinohe and Rockland, 2004).

The functional significance of the thalamocortical-corticocortical honeycomb is unknown, but could be related to differential processing by distinct postsynaptic populations. Apical dendrites of layer 2 

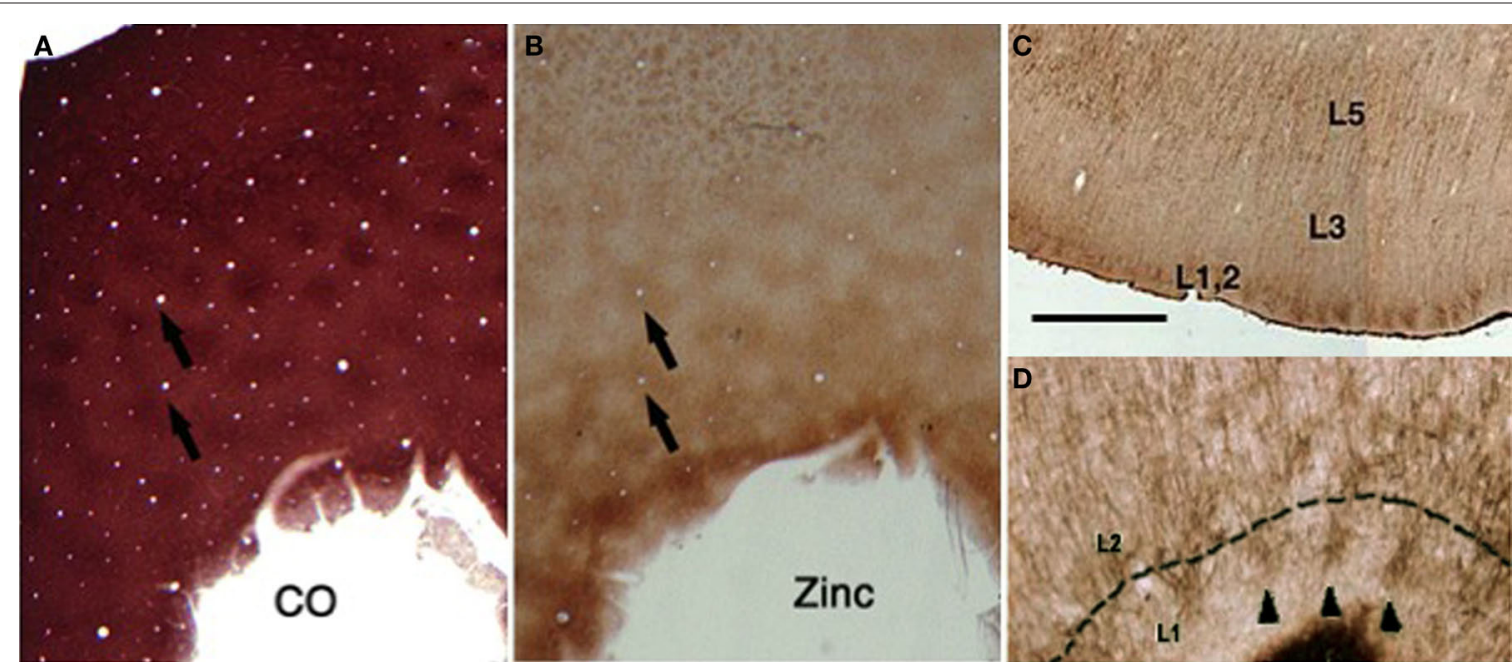

FIGURE 3 | Interdigitating systems. (A) Tangential section through monkey primary visual cortex, reacted for cytochrome oxidase (CO). Obvious patches correspond to thalamocortical terminations. (B) Adjacent section reacted for synaptic zinc, where patches correspond to a subset of corticocortical terminations. The top of the photo is cut tangential through layer 4A. The zinc-positive patches are complementary to the CO-patches in layer 3 (see arrows), and layer 4A. (C) Coronal section through the posterior orbitofrontal cortex of macaque, where MAP2 immunohistochemistry reveals distinct clusters of apical dendrites at the border of layers 1 and 2. These are likely to co-localize with zinc-positive terminations. (D) Higher magnification of C, where three dendritic clusters are indicated by arrowheads. (C) and (D) are modified from Figure 3 of Ichinohe and Rockland (2004). Scale bar in (C) = $1.0 \mathrm{~mm}, 160 \mu \mathrm{m}$ for (D), and 600 $\mu \mathrm{m}$ for (A) and (B). pyramids have been found to co-localize with zinc-positive cortical projections whereas those of layer 5 pyramids co-localize with the VGLUT-2 labeled thalamocortical projections (Ichinohe et al., 2003). Presumably, the two "streams" are integrated in a next step during local, intra- and inter-laminar processing. Why they need to be initially segregated is not clear.

The location of a honeycomb modularity at the border of layers 1 and 2 points to an independent role for this uppermost cortical stratum, distinguishable from that of layer 4 , and thus potentially a different system, embedded within a thalamic-based columnarity in layer 4 . Several recent reports have emphasized layer specificity on the basis of differential plasticity properties. A zone of distinctive structural plasticity, coinciding with layer 2 of mouse visual cortex, has been identified by pronounced remodeling of interneuron dendritic tips (Lee et al., 2008). Pharmacological blockade of cannabinoid receptors, also in mouse visual cortex, prevents the ocular dominance shift induced by monocular deprivation in the uppermost layers, but not in layer 4 (Liu et al., 2008).

\section{Interdigitation of cortical connections}

Both intrinsic and extrinsic cortical connections are often columnar in cross section. Despite decades of work, the organization of these connections, singly and in relation to each other, is only poorly understood. Global markers do not distinguish between intrinsic and extrinsic cortical projections, and double anterograde tracer injections may not be successful unless the appropriate placement within the target areas is achieved. Intracellular or juxtacellular injections potentially can display the total intrinsic and extrinsic arborizations for a single neuron or small number of neurons, but these techniques do not yield large numbers. Intracellular fills are often carried out in in vitro preparations, which are not suitable for long-distance extrinsic connections.
For extrinsic connections, in vivo injections of different anterograde tracers have identified two distinct patterns. In certain common cortical target areas, frontal and parietal projections were found to terminate in an array of interdigitating columns (in cross section); in others, the terminations had a laminar, not columnar complementarity (Selemon and Goldman-Rakic, 1988). A columnar termination pattern implies an initial segregation by laterally displaced populations of postsynaptic dendrites whereas laminar segregation implies, as in the hippocampus, segregation by depth along spatially intermingled dendritic populations.

\section{POINT 3}

Individual columns are embedded within distributed networks (Figures 4 and 5). Although column formation is often treated as a local event, columns might be best viewed as part of an interconnected network ("diaschisis"). This higher order connectivity is not easily accessible to experimentation, although it may become more so with the development of reliable transneuronal techniques. Intra-areal coordination of columnar architectures has been investigated during development by comparing layouts of orientation activity columns in areas V1 and V2 in 2-deoxyglucose experiments (Kaschube et al., 2009). One review of corticothalamic projections has proposed a "rule of parity." The authors propose to modify the idea of "reciprocity" to "parity," where the distribution of corticothalamic projections is seen as determined by convergence with branching patterns of prethalamic afferents (Deschenes et al., 1998).

Distributed organization occurs at several levels. First, cortical projection neurons have intrinsic collaterals. Intrinsic collaterals, as demonstrated most clearly by intracellular injections, extend 2-3 mm from the soma, and for neurons in layers 3 and 5, form multiple patches of terminations $(d \sim 250-500 \mu \mathrm{m})$ in monkey and cats (Gilbert and Wiesel, 1983; McGuire et al., 1991; Ojima and Jones, 1991; Yabuta 


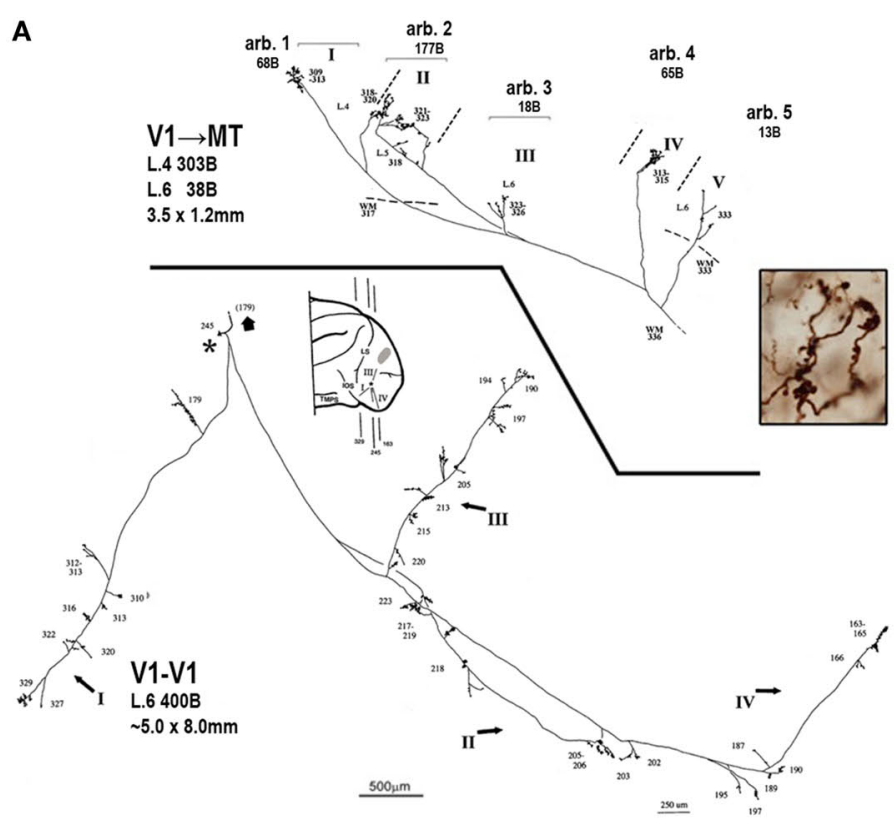

B

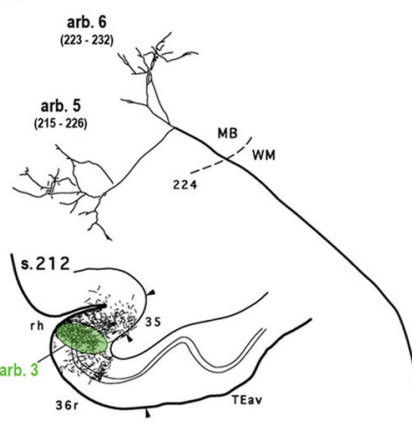

axon 4-4
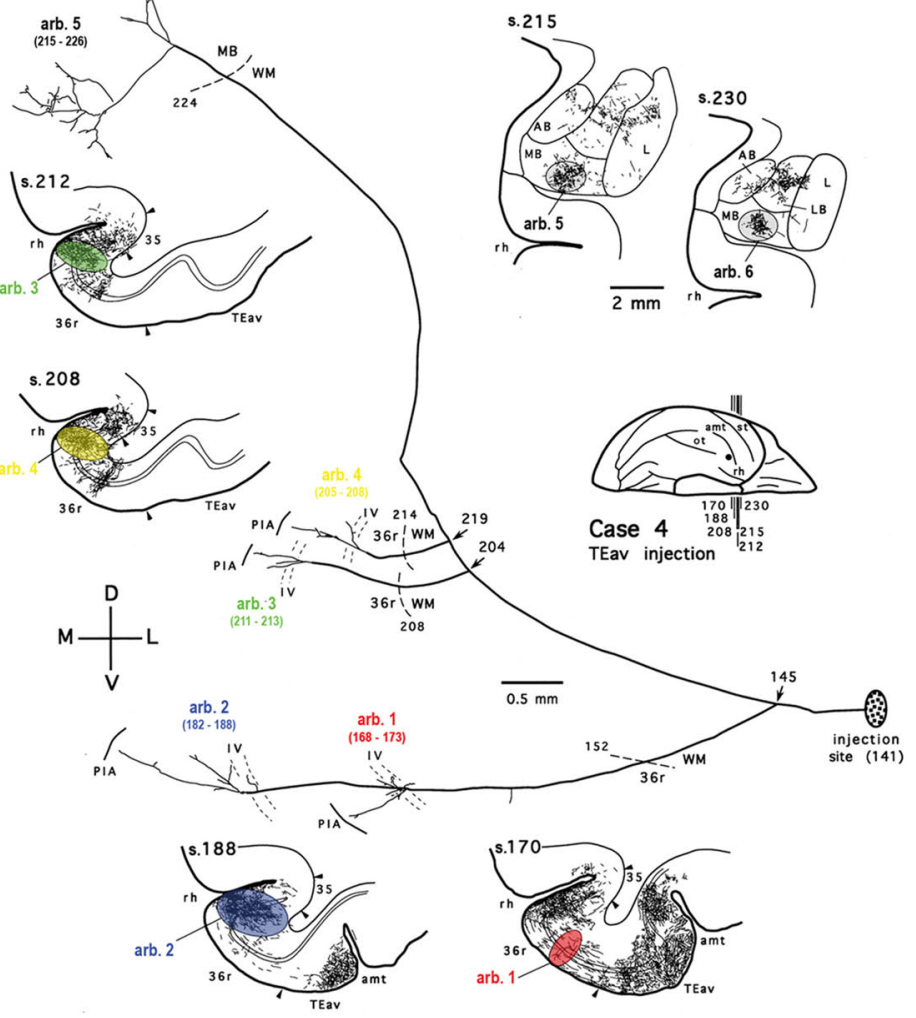

FIGURE 4 | Columns are part of distributed networks. (A)Top: Meynert cells in area $\mathrm{V} 1$ project to extrastriate area MT where they form multiple arbors in layers 4 and 6. One axon with five arbors (arb.) is illustrated. Large numbers = number of terminal specializations (boutons, b); and smaller numbers indicate individual tissue sections, where larger numbers are more anterior. Bottom: The same neurons form extensive intrinsic connections within area V1. For this neuron, four extended collaterals (I-IV) were identified. The anterior-posterior position of three coronal sections is indicated by lines on the schematic of the posterior half of the cerebral hemisphere, and the approximate position of the collaterals is indicated by I-IV. A BDA injection site is indicated by the shaded oval. Terminal specializations in MT are illustrated in the inset. Modified from Figure 1 of Rockland (2002). (B) A neuron anterogradely labeled by an injection of PHA-L in macaque area TEav has four arbors in adjoining parts of area 36, and two additional arbors in the amygdala. Arbors were followed to denser projection patches (color-coded in selected coronal sections) in different sections. Sections correspond to the numbered lines on the schematic of the monkey hemisphere. Modified from Figure 12 of Cheng et al. (1997). 

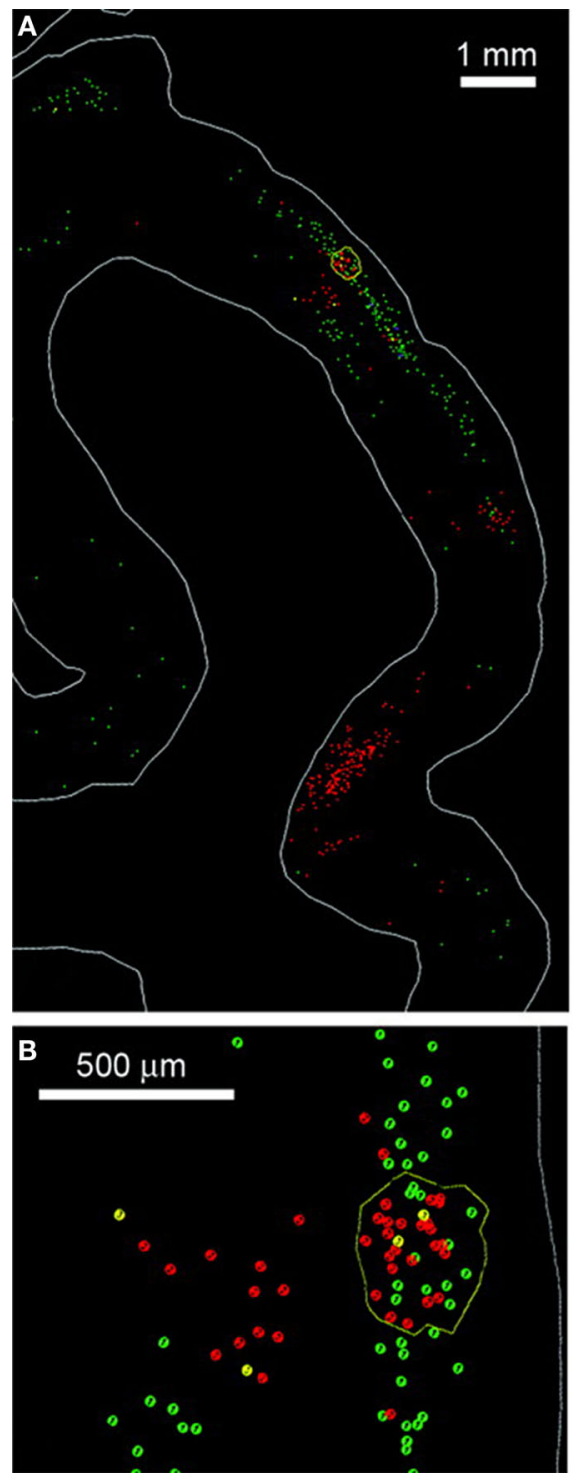

FIGURE 5 | Distributed terminations shown by retrograde tracers. (A) Small injections of two retrograde tracers in monkey temporal cortex result in large patches of red or green projection neurons, which converge to the respective injection sites. (B) Schematic of the monkey right hemisphere with the two injections (cholera toxin subunit B conjugated with alexa 488 (green

fluorescence) or alexa 555 (red fluorescence). The line indicates the level of the coronal section illustrated in (A). (C) Small clusters occur where single-colored

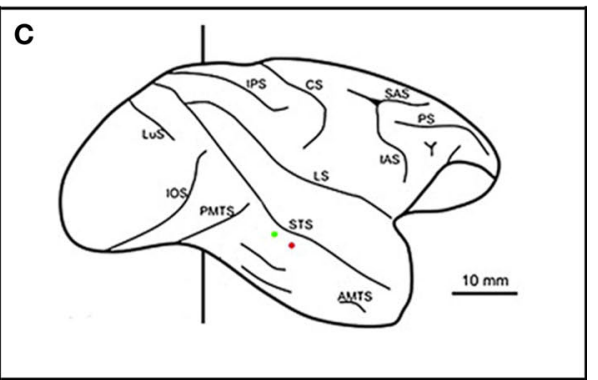

\section{D}

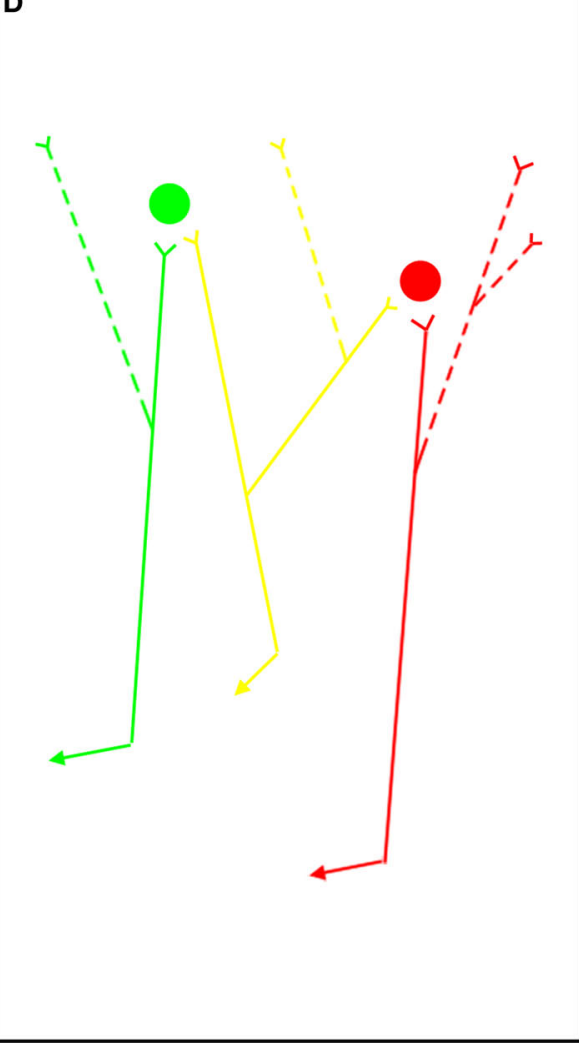

neurons are intermixed and where there are also double-labeled neurons. (D) The interpretation, consistent with analysis of anterogradely labeled single axons, is that neurons have branched arbors. Three neurons are represented schematically by colored triangles, and their branched axons by corresponding colored lines. Only some of the arbors (solid lines) will be labeled by a given injection, while others will fall outside the injected area (dashed lines). Modified from Figure 5, Borra et al. (2010). and Callaway, 1998). Terminations are preferentially concentrated in certain layers, and are thus more strictly "patchy" than "columnar. The number and spacing of terminal patches is documented for only a small number of pyramidal neurons, so that the degree of variability is unclear. Neurons in layer 6 have local collaterals, but these do not typically form patches (Anderson et al., 1993).

Second, extrinsically projecting axons typically have 2-4 arbors, each about $200-400 \mu \mathrm{m}$ in diameter, which distribute over a $2 \times 3 \mathrm{~mm}$ hollow space within the target area. Current thinking on columnar organization would predict that the multiple arbors innervate columns with similar response properties. However, the multiple arbors are not stereotyped, but are heterogeneous, possibly having different postsynaptic targets and synaptic effects. One arbor is often identifiable as "principal," on the basis of size and number of boutons (Zhong and Rockland, 2003), and could potentially have different, and even opposite effects from the secondary arbors. For example, axons projecting from V1 to extrastriate area MT in macaque typically have two spatially separated arbors to layer 4 (>1.0 mm apart), and another spatially offset arbor in layer 6 (Figure 4). If, as has been proposed, area MT has a compartmental organization according to directionality preference, the two layer 4 arbors might target two functionally similar columnar domains, 
but with functionally different consequences, while the intervening arbor in layer 6 could be within a functionally dissimilar domain (Rockland, 1989).

Third, a single axon can have branched collaterals to multiple cortical areas (Cheng et al., 1997; Mitchell and Macklis, 2005). How are the multiple projection foci, widely separated in space, determined? This is unknown, but one can think of piriform cortex, where odorants appear to be represented by unique and distributed ensembles of neurons, modeled as random convergent excitatory inputs from the olfactory bulb (Stettler and Axel, 2009). A not dissimilar organization may exist in sensory and association cortices, with respect to distributed ensembles of neurons, even though it has become more customary to assume a significantly contrasting organization for neocortex (Figures 4 and 5$)$.

\section{POINT 4}

Columns are not obligatory to cortex (Figure 6). In piriform (olfactory) cortex and other limbic areas, layer 1 is a major input layer, and layer 4 is either absent or poorly developed (Neville and Haberly, 2004). In these areas, it is layer 1 that has a complex organization; but this is in the tangential dimension, where layers $1 \mathrm{a}, 1 \mathrm{~b}$, and $1 \mathrm{c}$ are connectionally distinguishable strata. An interesting possibility is that features of "older," non-layer 4 cortex may be incorporated or embedded within neocortex. These

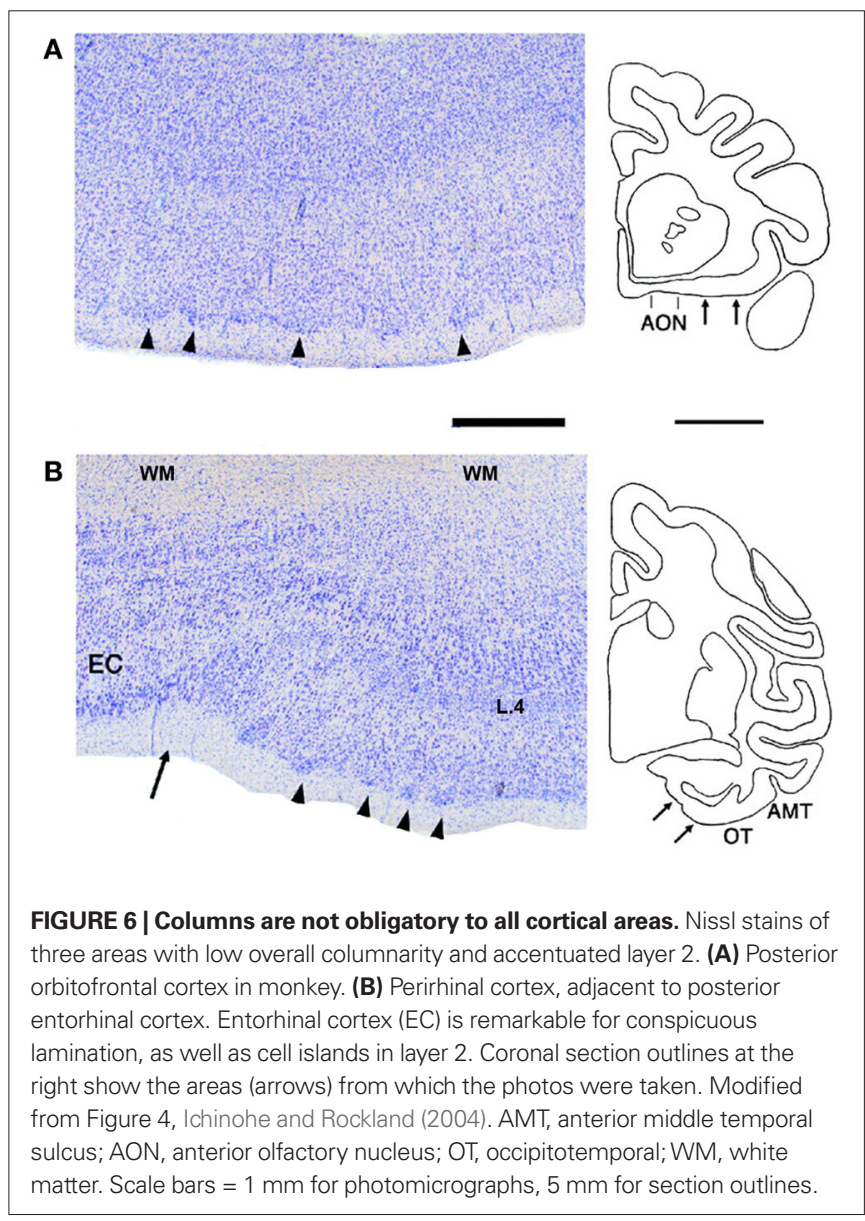

features would include a lack of modularity, at least in layer 1, and a heightened importance for apical dendritic tufts of underlying pyramidal cells.

The entorhinal cortex has prominent cell islands ("modules"), but these are confined to layer 2 . The presubiculum has pronounced cell islands, again confined to the upper layers. These co-localize or interdigitate with patches visualized by several neurochemical markers (Ding and Rockland, 2001). In the presubiculum, the development of the patchy organization has been attributed to reelin secreted by Cajal-Retzius cells, seemingly cooperating with the influences of early serotonergic projections (Nishikawa et al., 2002; Janusonis et al., 2004).

Comparative anatomy provides many examples of cortex apparently without anatomical columns or with dramatically modified columns. Whales and dolphins have a cortex with a small layer 4 and enlarged layer 1 (Hof and Van der Gucht, 2007). Connectional data are not available, but the prediction would be of a divergent, layer-1 dominant architecture, resembling that of piriform cortex in rodents. In the same species, the insular cortex has distinct cellular modules, but these are concentrated in layer 2 , similar to the general pattern in primate and rodent entorhinal cortex (layer 2) or rodent barrel cortex (layer 4) (Manger et al., 1998). The occipital cortex in giraffe has distinct cellular modules, but these are again concentrated in islands in layer 2 (DeFelipe, 2005).

Finally, cortical architecture can be significantly disrupted and yet apparently remain functionally intact. The disrupted barrel cortex in the reeler and in other mutant or transgenic mice is not associated with marked somatosensory deficits. The degree to which cortex is modifiable, and by what mechanisms, has been extensively investigated under various environmental manipulations. With the development of finer techniques, one can anticipate a new generation of genetic and molecular manipulations. Overexpression of NT3 is reported to result in an enhanced expression of dendritic bundles ("minicolumns") in rat barrel cortex (Miyashita et al., 2010).

\section{POINT 5}

Columns outside cerebral cortex (Figure 7). While vertical "columns" of terminations or projection neurons have been considered hallmarks of cortical structure, modularity per se is a common organizational principle in the brain. The periaqueductal gray contains longitudinal columns of afferent inputs, output neurons and intrinsic interneurons thought to co-ordinate different strategies for coping with different types of aversive stimuli (Bandler and Shipley, 1994; Keay and Bandler, 2001). The lateral septal nucleus is reported to have a complex system of chemically and connectionally distinct zones of transverse sheets (Risold and Swanson, 1998). Some thalamic nuclei have distinct domains, which are neurochemically and connectionally distinguishable (Rausell and Jones, 1991). The basal ganglia are organized into neurochemically and connectionally distinct striosomes and matrix (Graybiel and Ragsdale, 1978).

The superior colliculus (SC), a laminated subcortical structure, has an intricate tiered and mosaic modularity in the intermediate layers (Illing and Graybiel, 1986; Harting et al., 1992). These have been conveniently referenced to $200-600 \mu \mathrm{m}$ wide patches 


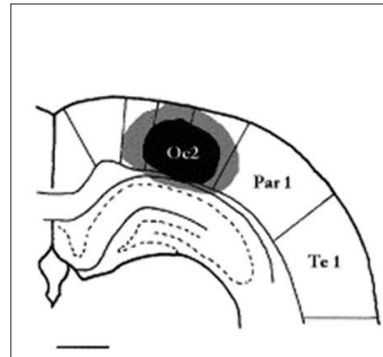

Interaural A 4.7

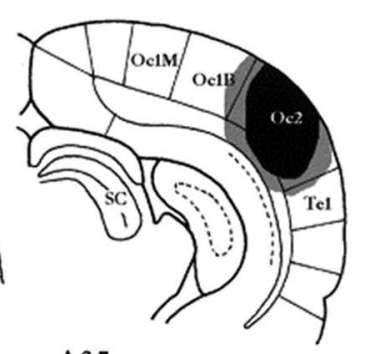

A 2.7

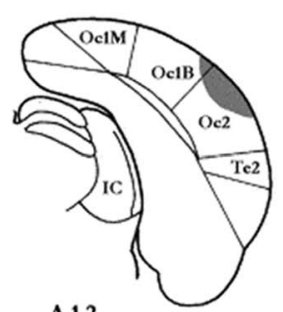

A 1.2

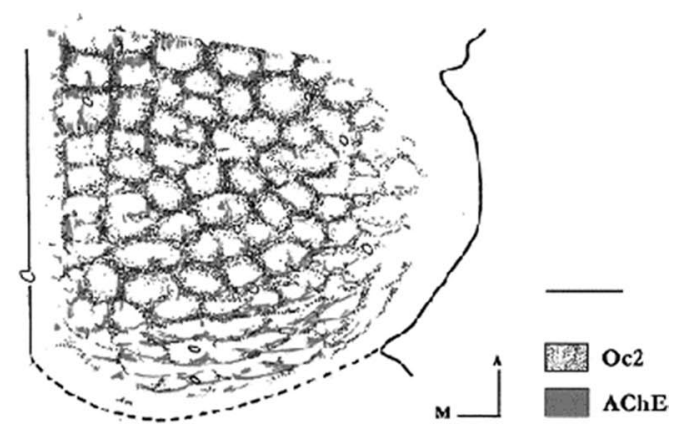

FIGURE 7 | Columns, as "modules", exist outside cerebral cortex. Three coronal sections (rat) to illustrate injection of WGA-HRP in visual cortex. This results (at right, tangential view of the superior colliculus) in a honeycomb pattern of cortical terminations (Oc2), which overlaps with a similar pattern shown by histochemistry for acetylcholinesterase (AChE). A, anterior; m, medial. Modified from Figure 1 from Mana and Chevalier, 2001. Scale bars $=1 \mathrm{~mm}$ for section outlines; $500 \mu \mathrm{m}$ for tangential colliculus. of acetylcholinesterase reactivity, corresponding to cholinergic inputs from the pedunculopontine nucleus (Harting et al., 1992; Mana and Chevalier, 2000). Both cortical and subcortical inputs contribute to the organization. AChE rich zones, predominantly in the dorsal SC, co-localize with nigrotectal and frontotectal inputs (i.e., motor-related), whereas somatosensory cortical and trigeminotectal projections (i.e., sensory-related), themselves overlapping, target the AChE reduced zones.

More recent investigations of collicular geometry have demonstrated that the neurochemical and input patches form a 3-D honeycomb lattice. This has been modeled as a high resolution matrix for the generation of directed orienting movements, where the multimodal nature of objects in the environment necessitate a complex array of multiple channels (Mana and Chevalier, 2001). A relatively constant number of 80-100 AChE compartments has been identified in a cross-species comparative study; and this has been taken to indicate a common genetic program.

In the cerebellar cortex, an elaborate array of modular subdivisions is revealed by histochemical markers, the topography of afferent projections and some efferent projections, and by gene expression in subpopulations of Purkinje cells (Voogd and Glickstein, 1998; Sillitoe and Joyner, 2007). Zebrin II expression reveals a parasagittal pattern of Purkinje cell stripes (PC), each consisting of a few hundreds to thousands of PCs, that is highly reproducible, activity independent, and conserved across species. Other molecular and connectivity markers have an orderly relation to zebrin + or zebrin-stripes (Larouche and Hawkes, 2006). The functional importance of this striking organization remains to be elucidated, but, similar to the mosaicism of the superior colliculus, has been suggested to subserve a massively parallel architecture with a high number of processing channels (Larouche and Hawkes, 2006). By contrast, the number of markers revealing a stripe-like organization in the cerebral cortex, at least at present, is limited.

\section{CONCLUSION}

As a term, column is imperfect. The word inevitably has connotations of something solid, repetitive, and static; columns "of something." The common alternatives - "module" or "patch" or "domain" to varying degrees suffer from the same problem. None succeed in conveying the fluid and dynamic properties which may more properly be quintessential cortical attributes.

With the acquisition of more detailed data combined with an openness to new interpretations and models, "column" may follow something of the evolution of "gene." At one point equated with an indivisible, discrete unit of genetic transmission, "gene" now implies an interleaved continuum of coding and regulatory information, where gene expression is controlled by combinatoric actions of transcription factors and other regulatory proteins (Gerstein et al., 2007; Mattick et al., 2009). An equivalent semantic expansion for "column" might come about when we have more information about types of columns and the range of operations. Are there "regulatory" columns? "silent" columns?

Progress can be expected to come from better characterization of cell types (Brown and Hestrin, 2009). This could take us beyond macrocolumns, which are now often visualized by global patterns and averaged connectivity. Re-examination would also be helped by full consideration that small areas of cortex may use different rules for connectivity and the associated columnarity. In a recent report, small injections of two retrograde tracers in monkey inferotemporal cortex appeared to result, in downstream visual areas, in small clusters with an elevated percentage of double-labeled neurons. This would correspond to clusters of more highly collateralized neurons or neurons with preferential connectivity to the two injection sites, as opposed to one of these (Borra et al., 2010).

Unfortunately, there is no easy alternative to "column," and no more specific terminology. But, perhaps under the influence of new data, the word and concept can change, so that the connotation is not crystalline, static, and repetitive, but more dynamic and variegated. For now, best may be to continue using the term, but more and more with something in mind that is closer to Sherrington's "enchanted loom," except that both structure as well as function should be imagined as in a dynamically fluid state.

\section{ACKNOWLEDGMENTS}

I would like to thank Dr. Nori Ichinohe for discussion, especially about Figure 5, and Naema Nayyar for help with manuscript preparation. 


\section{REFERENCES}

Adams, D. L., Sincich, L. C., and Horton, J. C. (2007). Complete pattern of ocular dominance columns in human primary visual cortex. J. Neurosci. 26, 10391-10403.

Alloway, K. D. (2008). Information processing streams in rodent barrel cortex: the differential functions of barrel and septal circuits. Cereb. Cortex 18, 979-989.

Anderson, J. C., Martin, K. A. C., and Whitteridge, D. (1993). Form, function, and intracortical projections of neurons in the striate cortex of the monkey Macaca nemistrinus. Cereb. Cortex 3, 412-420.

Bandler, R., and Shipley, M. T. (1994). Columnar organization in the midbrain periaqueductal gray: modules for emotional expression? Trends Neurosci. 17, 379-389.

Blasdel, G. G., and Lund, J. S. (1983). Termination of afferent axons in macaque striate cortex. J. Neurosci. 3, 1389-1413.

Borra, E., Ichinohe, N., Sato, T., Tanifuji, M., and Rockland, K. S. (2010) Cortical connections to area TE in monkey: hybrid modular and distributed organization. Cereb. Cortex 20, 257-270.

Brown, S. P., and Hestrin, S. (2009). Celltype identity: a key to unlocking the function of neocortical circuits. Curr. Opin. Neurobiol. 19, 415-421.

Cheng, K, Saleem, K. S., and Tanaka, K. (1997). Organization of corticostriatal and corticoamygdalar projections arising from the anterior inferotemporal area TE of the macaque monkey: a Phaseolous vulgaris leucoagglutinin study. J. Neurosci. 17, 7902-7925.

DeFelipe, J. (2005). "Reflections on the structure of the cortical minicolumn," in Neocortical Modularity and the Cell Minicolumn, ed.M.F. Casanova (New York: Nova Biomedical Books), pp. 57-92.

Deschenes, M., Veinante, P., and Zhang, Z. W. (1998). The organization of corticothalamic projections: reciprocity vs. parity. Brain Res. Rev. 28, 286-308.

Ding, S.-L., and Rockland, K. S. (2001). Modular organization of the monkey presubiculum. Exp. Brain Res. 139, 255-265.

Dyck, R. H., Chaudhuri, A., and Cynader, M. S. (2003). Experience-dependent regulation of the zincergic innervation of visual cortex in adult monkeys. Cereb. Cortex 13, 1094-1109.

Freund, T. F., Martin. K. A., Soltesz, I., Somogyi, P., and Whitteridge, D. (1989). Arborisation pattern and postsynaptic targets of physiologically identified thalamocortical afferents in striate cortex of the macaque monkey. J. Comp. Neurol. 289, 315-336.
Gerstein, M. B., Bruce, C., Rozowsky, J. S., Zheng, D., Du, J., Korbel, J. O., Emanuelsson, O., Zhang, Z. D., Weissman, S., and Synder, M. (2007). What is a gene, post-ENCODE? History and updated definition. Genome Res. 17, 669-681.

Gilbert, C. D., and Wiesel, T. N. (1983). Clustered intrinsic connections in cat visual cortex. J. Neurosci. 3, 1116-1133.

Graybiel, A. M., and Ragsdale, C. W. (1978). Histochemically distinct compartments in the striatum of humans, monkeys, and cat demonstrated by acetylthiocholinesterase staining. Proc. Natl. Acad. Sci. USA 75, 5723-5726.

Harris, R. M., and Woolsey, T. A. (1979). Morphology of Golgi-impregnated neurons in mouse cortical barrels following vibrissae damage at different post-natal ages. Brain Res. 161 143-149.

Harting, J. K., Updyke, B. V., and Lieshout, D. P. V. (1992). Corticotectal projections in the cat: anterograde transport studies of twenty-five cortical areas. J. Comp. Neurol. 324, 379-414.

Hof, P. R., and Van der Gucht, E. (2007). Structure of the cerebral cortex of the humpback whale, Megaptera novaeangliae (Cetacea, Mysticeti, Balaenopteridae). The Anat. Rec. 290, $1-31$.

Horton, J. C., and Adams, D. L. (2005). The cortical column: a structure without a function. Philos. Trans. R. Soc. Lond., B, Biol. Sci. 360, 837-862.

Horton, J. C., and Hocking, D. R. (1996) Intrinsic variability of ocular dominance column periodicity in normal macaque monkeys. J. Neurosci. 16, 7228-7239.

Horton, J. C., and Hocking, D. R. (1998) Monocular core zones and binocular border stripes in primate striate cortex revealed by contrasting effects of enucleation, eyelid suture, and retinal laser lesions on cytochrome oxidase activity. J. Neurosci. 15, 5433-5455.

Hubener, M., and Boltz, J. (1992). Relationships between dendritic morphology and cytochrome oxidase compartments in monkey striate cortex. J. Comp. Neurol. 324, 67-80.

Ichinohe, N., and Rockland, K. S. (2004) Region specific micromodularity in the uppermost layers in primate cerebral cortex. Cereb. Cortex 14 1173-1184.

Ichinohe, N., Fujiyama, F., Kaneko, T., and Rockland, K. S. (2003). Honeycomblike mosaic at the border of layers 1 and 2 in the cerebral cortex. J. Neurosci. $23,1372-1382$

Illing, R.-B., and Graybiel, A. M. (1986) Complementary and non-matching afferent compartments in the cat's superior colliculus: innervation of the acetycholinesterase-poor domain of the intermediate zone. Neuroscience 18, 373-394.

Imig, T. J., and Reale, R. A. (1981) Ipsilateral corticocortical projections related to binaural columns in cat primary auditory cortex. J. Comp. Neurol. 203, 1-14.

Inan, M., and Crair, M. C. (2007) Development of cortical maps: perspectives from the barrel cortex. Neuroscientist 13, 49-60.

Janusonis, S., Gluncic, V., and Rakic, P. (2004). Early serotonergic projections to Cajal-Retzius cells: relevance for cortical development. J. Neurosci. 24, 1652-1659.

Jones, E. G. (2000). Microcolumns in the cerebral cortex. Proc. Natl. Acad. Sci. USA 97, 5019-5021.

Kaschube, M., Schnabel, M., Wolf, F., and Lowel, S. (2009). Interareal coordination of columnar architectures during visual cortical development. Proc. Natl. Acad. Sci. USA 106, 17205-17210.

Katz, L. C., Gilbert, C. D., and Wiesel, T. N. (1989). Local circuits and ocular dominance columns in monkey striate cortex. J. Neurosci. 9, 1389-1399.

Keay, K.A., and Bandler, R. (2001). Parelle circuits mediating distinct emotional coping reactions to different kinds of stress. Neurosci. Biobehav. Rev. 25 , 669-678.

Krieger, P., Kuner, T., and Sakmann, B. (2007). Synaptic connections between layer $5 \mathrm{~B}$ pyramidal neurons in mouse somatosensory cortex are independent of apical dendritic bundling. $J$. Neurosci. 27, 11473-11482.

Land, P. W., and Erickson, S. L. (2005) Subbarel domains in rat somatosensory (S1) cortex. J. Comp. Neurol. 490, 414-426.

Larouche, M., and Hawkes, R. (2006) From clusters to stripes: the developmental origins of adult cerebellar compartmentation. Cerebellum 5, 77-88.

Lee, W. C., Chen, J. L., Huang, H., Leslie, J. H., Amitai, J., So, P. T., and Nedivi, E. (2008). A dynamic zone defines interneuron remodeling in the adult neocortex. Proc. Natl. Acad. Sci. USA, 105, 19968-19973.

LeVay, S., Hubel, D. H., and Wiesel, T. N. (1975). The pattern of ocular dominance columns in the macaque visual cortex, revealed by a reduced silver stain. J. Comp. Neurol. 159, 559-576.

Liu, C.-H., Heynen, A. J., Shuler, M. G. H., and Bear, M. F. (2008). Cannabinoid receptor blockade reveals parallel plasticity mechanisms in different layers of mouse visual cortex. Neuron 58 , 340-345.

Malach, R. (1994). Cortical columns as devices for maximizing neuronal diversity. Trends Neurosci. 17, 101-104.

Mana, S., and Chevalier, G. (2000). Honeycomb-like structure of the intermediate layers of the rat superior colliculus, with additional observations in several other mammals: AChE patterning. J. Comp. Neurol. 419, 137-153.

Mana, S., and Chevalier, G. (2001). Honeycomb-like structure of the intermediate layers of the rat superior colliculus: afferent and efferent connections. Neuroscience 103 673-693.

Manger, P., Sum, M., Szymanski, M., Ridgway, S., and Krubitzer, L. (1998). Modular subdivisions of dolphin insular cortex: does evolutionary history repeat itself? J. Cog. Neurosci. 10, 153-166.

Markram, H. (2008). Fixing the location and dimensions of functional neocortical columns. HFSP J. 2, 132-135.

Mattick, J. S., Taft, R. J., and Faulkner, G. J. (2009). A global view of genomic information-moving beyond the gene and the master regulator. Trends Genet. 26, 21-28.

McGuire, B. A., Gilbert, C. D., Rivlin, P. K., and Wiesel, T. N. (1991). Targets of horizontal connections in macaque primary visual cortex. J. Comp. Neurol. 305, 370-392.

Mitchell, B. D., and Macklis, J. D. (2005). Large-scale maintenance of dual projections by callosal and frontal projection neurons in adult mice. J. Comp. Neurol. 482, 17-32.

Miyashita, T., Wintzer, M., Kurotani, T., Konishi, T., Ichinohe, N., and Rockland, K. S. (2010). Neurotrophin-3 is involved in the formation of apical dendritic bundles in cortical layer 2 of the rat. Cereb. Cortex 20, 229-240.

Neville, K. R., and Haberly, L. B. (2004). "Olfactory cortex," in The Synaptic Organization of the Brain, ed. G. M. Shepherd (New York: Oxford University Press), pp. 415-454.

Nishikawa, S., Goto, S., Hamasaki, T., Yamada, K., and Ushio, Y. (2002). Involvement of reelin and CajalRetzius cells in the developmenta formation of vertical columnar structures in the cerebral cortex: evidence from the study of mouse presubicular cortex. Cereb. Cortex $12,1024-1030$.

Ojima, H., and Jones, E. G. (1991). Patterns of axonal collateralization of identified supragranular pyramidal neurons in the cat auditory cortex. Cereb. Cortex $1,80-94$.

Otsuka, T., and Kawaguchi, Y. (2008). Firing-pattern-dependent specificity of cortical excitatory feed-forward subnetworks. J. Neurosci. 28, 86-95. 
Peters, A., Cifuentes, J. M., and Sethares, C. (1997). The organization of pyramidal cells in area 18 of the rhesus monkey. Cereb. Cortex 7, 405-421.

Pucak, M. L., Levitt, J. B., Lund, J. S., and Lewis, D.A. (1996). Patterns of intrinsic and associational circuitry in monkey prefrontal cortex. J. Comp. Neurol. 376, 614-630.

Rausell, E., and Jones, E. G. (1991). Chemically distinct compartments of the thalamic VPM nucleus in monkeys relay principal and spinal trigeminal pathways to different layers of the somatosensory cortex. J. Neurosci. 11, 226-237.

Rice, F. L. (1995). “Comparative aspects of barrel structure and development," in Cerebral Cortex: The Barrel Cortex of Rodents, Vol. 11, eds. A. Peters, E. G. Jones, and I. T. Diamond (New York: Plenum Press), pp. 1-76.

Risold, P. Y., and Swanson, L. W. (1998). Connections of the rat lateral septal complex. Brain Res. Rev. 24, 115-195.

Rockland, K. S. (1989). Bistratified distribution of terminal arbors of individual axons projecting from area $\mathrm{V} 1$ to middle temporal area (MT) in the macaque monkey. Vis. Neurosci. 3, 155-170.
Rockland, K. S. (2002). Non-uniformity of extrinsic connections and columnar organization. J. Neurocytol. 31, 247-253.

Rockland, K. S., and Drash, G. (1996). Collateralized divergent feedback connections that target multiple cortical areas. J. Comp. Neurol. 373, 529-548.

Rockland, K. S., and Ichinohe, N. (2004). Some thoughts on cortical minicolumns. Exp. Brain Res. 158, 265-277.

Rockland, K. S., and Pandya, D. N. (1979). Laminar origins and terminations of cortical connections of the occipital lobe in the rhesus monkey. Brain Res. 179, 3-20.

Rockland, K. S., Andresen, J., Cowie, R. J., and Robinson, D. L. (1999). Single axon analysis of pulvinocortical connections to several visual areas in the macaque. J. Comp. Neurol. 406, 221-250.

Roney, K. J., Scheibal, A. B., and Shaw, G. L. (1979). Dendritic bundles: survey of anatomical experiments and physiological theories. Brain Res. Rev. 1, 225-271.

Sato, T. R., Gray, N. W., Mainen, Z. F., and Svoboda, K. (2007). The functional microarchitecture of the mouse barrel cortex. PLoS Biol. 5, e189 doi: 10.1371/ journal.pbio.0050189.

Selemon, L. D., and Goldman-Rakic, P. S. (1988). Common cortical and subcortical targets of the dorsolateral prefrontal and posterior parietal cortices in the rhesus monkey: evidence for a distributed neural network subserving spatially guided behavior. $J$. Neurosci. 8, 4049-4068.

Sillitoe, R. V., and Joyner, A. L. (2007) Morphology, molecular codes, and circuitry produce the three-dimensional complexity of the cerebellum. Annu. Rev. Cell Dev. Biol. 23 549-577.

Stettler, D. D., and Axel, R. (2009). Representation of odor in the piriform cortex. Neuron 63, 854-864.

Takahata, T., Higo, N., Kaas, J. H., and Yamamori, T. (2009). Expression of immediate-early genes reveals functional compartments within ocular dominance columns after brief monocular inactivation. Proc. Natl. Acad. Sci. USA 106, 12151-12155.

Tanaka, K. (2003). Columns for complex visual object features in the inferotemporal cortex: clustering of cells with similar but slightly different stimulus selectivities. Cereb. Cortex 13, 90-99.

Voogd, J., and Glickstein, M. (1998). The anatomy of the cerebellum. Trends Neurosci. 21, 370-375.

Yabuta, N.H., and Callaway, E. M. (1998). Cytochrome-oxidase blobs and intrinsic horizontal connections of layer $2 / 3$ pyramidal neurons in primate V1. Vis. Neurosci. 15, 1007-1027.

Yoshimura,Y., Dantzker,J.L., and Callaway, E. M. (2005). Excitatory cortical neurons form fine-scale functional networks. Nature 433, 868-873.

Zhong, Y.-M., and Rockland, K. S. (2003). Inferior parietal lobule projections to anterior inferotemporal cortex (area TE) in macaque monkey. Cereb. Cortex, 13, 527-540.

Conflict of Interest Statement: The author declares that the research was conducted in the absence of any commercial or financial relationships that could be construed as a potential conflict of interest.

Received: 18 February 2010; paper pending published:03 March 2010; accepted:06 May 2010; published online: 09 June 2010.

Citation: Rockland KS (2010) Five points on columns. Front. Neuroanat. 4:22. doi: 10.3389/fnana.2010.00022

Copyright (c) 2010 Rockland. This is an open-access article subject to an exclusive license agreement between the authors and the Frontiers Research Foundation, which permits unrestricted use, distribution, and reproduction in any medium, provided the original authors and source are credited. 